\title{
RESEARCH
}

\section{Towards Fidelity in Pharmacy Education with the Patient Care Process for Delivering Comprehensive Medication Management}

\author{
Catherine Cone, PharmD, Danielle Gundrum, PharmD, Martin S Lipsky, MD \\ Roseman University of Health Sciences, South Jordan, Utah \\ Corresponding Author: Danielle Gundrum, Roseman University of Health Sciences, 10920 S River Front Pkwy, South Jordan, UT \\ 84095. Tel: 801-878-1093. Email: dgundrum@ roseman.edu
}

Submitted January 28, 2021; accepted July 26, 2021; ePublished August 2021

\begin{abstract}
Objective. To evaluate fidelity to the Comprehensive Medication Management (CMM) framework using CMM essential functions to analyze the domains of content and competency in a patient care skills course.

Methods. This mixed methods approach compared the Pharmacist's Patient Care Process II (PPCP II) skills course curriculum to the nationally developed CMM framework. CMM curriculum content was mapped to the framework and the percentage of omissions and deficiencies were calculated. Student competency was analyzed using assessment scores in a competency-based learning model.

Results. For the content domain, CMM comprised 41.5 class hours (40.7\%) of the total 102-hour PPCP II course. CMM curriculum omissions and deficiencies were both calculated at $14.3 \%$, yielding an overall $71.4 \%$ alignment to the CMM framework. For the competency domain, the percentage of students initially achieving competence ranged from $76.6 \%$ to 98.7\% on formative assessments in 2018-2019 and 2019-2020. For the summative assessment, 87.5\% and 69.2\% respectively achieved competency on their first attempt with levels rising significantly to $98.8 \%$ and $98.7 \%$ after remediation. Overall, $98.7 \%$ achieved competency in presented CMM materials in this course.

Conclusion. This study found that about $70 \%$ of the CMM framework for the core domain of content can be covered and competency achieved by students in approximately 40 hours of direct curricular time. Identified omissions and deficiencies highlight opportunities for course improvement, and remediation of skills resulted in a significant improvement in the number of students achieving competence in CMM helping to promote fidelity in the profession of pharmacy.

Keywords: Comprehensive Medication Management; CMM; fidelity; remediation; pharmacy education
\end{abstract}

\section{INTRODUCTION}

Drs. Sorensen and colleagues issued a call in the spring of 2020 to teach the pharmacist patient care process with "greater consistency, specificity, and intentionality" using the comprehensive medication management (CMM) framework. ${ }^{1}$ The Joint Commission of Pharmacy Practitioners (JCPP) established the Pharmacists' Patient Care Process (PPCP) to help pharmacist's design, implement and monitor drug therapy. ${ }^{2} \mathrm{CMM}$ offers a more detailed and pharmacy specific process for educators and practicing pharmacists to implement PPCP in a consistent, predictable and measurable fashion. ${ }^{3}$ However, if the CMM framework is not thoroughly integrated and assessed in a curriculum, key components may be missed and educators may fail to consistently prepare students for practice. Livet and colleagues outline a design for fidelity assessment in implementing and practicing CMM that include three core elements: context, content and competence. ${ }^{4}$ In a practice setting, context is the adherence to the infrastructure needed to support CMM, content is the extent to which the pharmacist and team adhere to the CMM patient care process, and competence is the extent to which the pharmacist and team demonstrate skillful delivery of CMM. ${ }^{4}$

Applying CMM to an educational setting depends on its core elements. Context, or the environment a pharmacist practices CMM, usually aligns with social and administrative science courses that educate students about health systems or courses where setting up clinical services is taught and assessed. In contrast, content and competence for CMM fits better in skills courses where direct patient care is taught and assessed in a clinical skills lab.

The patient care process for delivering CMM provides a framework for the clinical skills of CMM (content) by delineating essential functions that a pharmacist needs to accomplish. ${ }^{3}$ Promoting fidelity in instruction at colleges and schools of pharmacy within the CMM framework is critically important to prepare students for the modern role of pharmacy in patient care. Fidelity refers to the adherence to the structure, implementation and degree of exactness to which a process can be reliably reproduced in a manner that achieves the original intent. ${ }^{5,6}$ Fidelity to the CMM framework promotes consistency, specificity, and intentionality in patient care and gives patients, other health care 
providers, insurance companies or other payers a clear set of expectations from the pharmacist care team member. ${ }^{1,3,4}$ To promote fidelity to CMM in education, pharmacy curriculum should promote competency of CMM essential functions (content) in the classroom, lab, and experiential rotations. This requires a process that maps learning activities, offers formative and summative assessments of learning and ensures student competency. This study evaluates curricular fidelity to the CMM framework in a dedicated patient care skill development course by analyzing CMM related to content and competence domains.

\section{METHODS}

This retrospective review was determined to be exempt by the Roseman University of Health Sciences (RUHS) Institutional Review Board (IRB). This mixed methods study evaluated a clinical skills lab course focusing on the core elements of content and competency. Content mapping utilized the CMM framework developed by the CMM in Primary Care Research Team ${ }^{3}$ to analyze curriculum through mapping. Analysis of student grades and passing rates formed the basis for assessment of competency.

\section{Setting and Study Population}

RUHS is a private, nonprofit health sciences university. The RUHS College of Pharmacy (COP) offers a threeyear accelerated PharmD program accredited by the Accreditation Council for Pharmacy Education (ACPE) as a single program administered on two separate and distinct campuses, one in Henderson, Nevada and one in South Jordan, Utah. The study sample consisted of a convenience sample of the 158 students comprising the 2018-19 and 2019-20 classes on the Utah campus. The PharmD program bases its curriculum on a competency-based learning model embedded in a block system. The program includes 72 weeks of didactic and skill-based instruction and 14 weeks of introductory pharmacy practice experiences (IPPEs) in the first two years of the program.

\section{Assessing Fidelity}

This study used fidelity of implementation to assess fidelity. Implementation can be roughly divided into two major categories: fidelity to structure ${ }^{7}$ characterized by the content domain and fidelity to process ${ }^{7}$ characterized by the competency domain. Criteria for fidelity to structure includes adherence defined as whether components are being delivered as designed, and duration defined as the number, length, or frequency of sessions implemented. ${ }^{5}$ To measure fidelity to structure, the study assessed adherence by mapping CMM essential functions to curriculum and to duration by assessing the hours devoted to CMM core elements (content domain). Fidelity to process includes criteria such as content delivery, quality and participant responsiveness. ${ }^{5}$ To assess process, the study examined the educational tools used to teach CMM elements and student competency of CMM (competency domain) as a marker of program effectiveness and participant responsiveness. Reproducibility is important to fidelity and the study used two years of test data to assess process reliability.

\section{Method One: Evaluation of Content Domain}

\section{Curriculum Structure}

Curriculum structure and documentation of faculty time spent teaching CMM provides perspective and assures reproducibility. The skills-based course, the Pharmacist's Patient Care Process II (PPCP II), was a required 5-credit course scheduled in the second and final didactic year and was where CMM was taught and assessed. PPCP II ran sequentially with IPPE's to incorporate direct patient care into the skills-based course to help cement learning. The PPCP II course consisted of 102 class hours (divided into four blocks each lasting between 24-30 hours) with an additional 16 hours built into the schedule for students requiring remediation. Table 1 outlines the PPCP II CMM curriculum which formed the basis of this analysis.

\section{Mapping (Alignment with CMM Framework)}

RUHS uses a competency-based learning model in which students must demonstrate competency with course objectives on assessments. The effectiveness of a competency-based learning model depends on courses developing and adhering to clearly stated objectives and assessing those objectives at a set level (in the case of RUHS at $90 \%$ for summative assessments of learning). CMM essential functions 1-5 constitute the CMM framework. The study used content mapping to determine the extent to which PPCP II curriculum addressed each essential function. Two authors (DG and CC) manually reviewed and mapped CMM activities, and formative and summative assessments of learning (Table 2). In cases of disagreement, the faculty members discussed the item until they reached consensus. 
The formative and summative assessment strategies in Table 2 were divided into separate categories. Progressive assignments in block one (see Table 1) built student knowledge and skills, leading to more complex skills assessments during blocks two and three. Formative assessments included a Medical Record based Subjective, Objective, Assessment and Plan (SOAP) Note and Problem List (PL) assignment, the Community based (IPPE patient) SOAP and PL project, and an Objective Structured Clinical Examination (OSCE) where students verbally presented a patient case. The summative assessment consisted of students interviewing a simulated patient with a medical record provided, and then developing a PL and SOAP note (termed OSCE Patient interview, SOAP note, \& PL) in block four.

\section{Evaluation of Curriculum and CMM Alignment}

Analysis of curriculum and alignment to CMM took two forms. First, PPCP II course data from two academic years 2018-19 and 2019-20 were used to develop descriptive statistics such as time spent teaching, learning, and assessing CMM. Second, alignment of the framework with course curriculum was utilized to uncover gaps and deficiencies. Omissions were defined as essential functions considered absent from the course and deficiencies were defined as essential functions that were present but not fully taught, developed or assessed. The study team who audited the PPCP II curriculum similarly studied curricular gaps to identify omitted and deficient curriculum. The gap analysis was used to calculate percentage alignment of CMM curriculum with the CMM framework. Alignment was calculated in the following manner. The five CMM essential functions and their operational subcategories (1a, 1b, 1c, etc.) totaled 29 elements. However, because of its complexity $1 \mathrm{~b}$ was subdivided into 13 additional components and combined with the other 29 elements to yield 42 distinct CMM elements. Percentages were calculated using the formula (omissions + deficiencies/ 42 total elements) X 100. To determine overall course alignment with CMM essential functions, the percentage of combined omissions and deficiencies was subtracted from 100.

\section{Method Two: Evaluation of Competency Domain}

Students demonstrated competency by scoring $80 \%$ or higher on formative assessments and $90 \%$ or higher on summative assessments of learning. If a student failed an assessment, they received feedback with an opportunity to retest and demonstrate competency. Students who failed their repeat assessment required summer remediation and had a third opportunity to demonstrate competency. Student competency was analyzed using the results of three formative and one summative assessments of learning. Analyses included calculating the mean percentage scores on the assessments, minimum and maximum scores, the percentage of students passing their first and, second assessments (second attempt), and reporting if any students failed to pass the course (third attempt). The percentage of students achieving overall competency from both years was also reported. A two-tailed, paired t-test was used to compare individual student scores from the summative assessment of learning on their first attempt and second test attempts.

\section{RESULTS}

\section{Content Domain}

Table 2 displays the mapping of CMM essential functions 1, 3, 4, and 5 to PPCP II curriculum. Essential function 2 is described separately in the next section. Instances where none of the PPCP II curriculum matched to an essential function (an omission) or when there was partial compliance (a deficiency) with an essential function are noted in Table 2 and described in greater detail in Table 3.

\section{CMM Essential Function 2 - Assess Information and Formulate a Medication Therapy Problem List}

Before students analyzed patient cases, they attended an Ordering Disease States and Setting Goals session (essential function $2 \mathrm{~g}$ - prioritize the patient's medication therapy problems). Students learned to identify indication ( $2 \mathrm{~b}$ ), effectiveness (2c), safety (2d), and adherence (2e), also known as IESA and disease related issues during the Development of a Problem List (PL) and the Collect, Assess, and Plan Activity sessions. Adherence, sometimes termed compliance is first introduced in the first-year skills lab. ${ }^{8,9}$ It is also taught and assessed during the PPCP II course and included in this analysis. For students to become competent in developing a problem list, they must learn to further refine the IESA components. In the Use of LexiComp(C) lecture, instructors teach students to assess drug-drug and drug-disease interactions, adverse reactions, and dosing. Students learned how to apply guidelines, to determine if a medication is indicated, to identify if it is first line, second line etc., and to individualize evidence-based treatment to a patient's goals in the Use of Guidelines lecture. Instructors also taught students to answer CMM essential function questions from the framework. A PL rubric helped students organize thought processes that address elements $2 \mathrm{a}$ (assess and prioritize the patient's active medical conditions considering clinical and patient goals of therapy) and $2 \mathrm{f}$ (formulate a medication therapy PL), and to incorporate IESA and disease related issues. Students also learned how to apply subjective and objective findings and to use patient findings to tailor treatment goals and plans (2a). The rubric and classroom 
discussions and activities promoted a student's ability to analyze information obtained by patient interview (during IPPE) and via the medical record (1c). In-class practice included two patient cases where students reviewed patient information and applied the appropriate guideline(s) and evidence.

Faculty used the PL rubric for additional activities during the remainder of the course. These include items outlined in Table 1 indicated by the word problem list or PL in the title. Students learned to transform PL elements into a SOAP note during the Problem-solving using PPCP/SOAP session and the Medical Record based SOAP Note \& PL assignment. Throughout the course, faculty guided students from novice learners to competence by promoting higher order thinking and evidence-based practices. For example, faculty members asked students to present a rationale for their patient treatment recommendation(s) for each disease state so faculty could assess their rationale and probe student thought processes. Additionally, to promote evidence-base practice students needed to cite evidence-based sources. Patient and medication assessment (A in SOAP) included an analysis of the recommended goals of therapy, PL issues identified, subjective or objective findings, as well as lifestyle.

\section{Evaluation of Curriculum and Mapping}

Table 1 summarizes the CMM curriculum in the PPCP II course. CMM comprised 41.5 class hours $(40.7 \%)$ of the total 102-hour course. These hours break down as follows: faculty time presenting CMM in class ( 8.3 hours or $8.1 \%$ of course), student class time spent on learning activities (9.2 hours or 9\%), student time spent on formative assessment (18 hours or $17.6 \%$ ), and student time spent on summative assessment (6 hours or 5.9\%). Table 3 compares curriculum to essential functions and identified six missing areas (omissions) and six inadequately addressed areas (deficiencies). The percentage of omitted essential functions and the percentage of deficient essential functions were $14.3 \%(6 / 42 * 100)$ each, resulting in a $71.4 \%$ alignment of the course to CMM.

\section{Competency Domain}

Tables 4 and 5 present an analysis of student competency of CMM-related curriculum over the course of two academic years (2018-19 and 2019-20). A total of three formative assessments and one summative assessment were used to evaluate whether a student achieved CMM competency. In both academic year cohorts, between $76.6 \%$ and $98.7 \%$ of students achieved competency on the three formative assessments of learning activities on their first attempt. This increased to $98.7 \%$ and $100 \%$ after retesting (second attempt). Only one student in each academic year required summer remediation (third attempt). Similar results were seen with the summative assessment where competency was demonstrated on initial testing by $87.5 \%$ of students in the $2018-19$ academic year and $69.2 \%$ in the $2019-20$ academic year. After reassessment, overall competency levels increased to $98.8 \%$ and $98.7 \%$ respectively, with only one student in each academic year requiring summer remediation. These students also failed to achieve competency during summer remediation but successfully repeated the course the following academic year. In the end, $98.7 \%$ of students in both academic years combined achieved competency on the summative assessment of learning by the end of the course. Summative test scores significantly improved following remediation and reassessment.

\section{DISCUSSION}

The Accreditation Council for Pharmacy Education (ACPE) 2016 standards mandated that all pharmacy schools prepare students to deliver patient care using the PPCP framework. ${ }^{8}{ }^{80}$ PPCP's five-step framework includes collect, assess, plan, implement, and follow-up. ${ }^{2}$ However, these steps are not unique to pharmacy. ${ }^{11-13}$ Thus, Sorenson and colleagues outlined a framework of "essential functions" that define PPCP and provide operational definitions for implementation to prepare students for the pharmacist's unique role in patient care. ${ }^{1,3}$ This helps assure that PPCP is taught, learned, and applied in a manner that consistently produces the intended outcome. By explicitly defining the essential functions and outlining a process for pharmacists to operationalize PCPP, CMM helps ensure fidelity to the process..$^{3}$

CMM offers a "whole person" method that requires an individualized patient approach using evidence-based management that incorporates each patient's overall condition, history, comorbidities and unique needs as well as their medications leading to a more advanced, coordinated level of pharmacy care. While other papers described and evaluated how schools incorporate PPCP into their curricula, ${ }^{14-18}$ this study examined the degree to which the CMM educational materials taught and assessed in a skills-based course aligned with the CMM framework (content) and the degree to which the pharmacy students in the course demonstrated competency with CMM (competence). This study adds to the literature by being the first to our knowledge to use the CMM framework to explore student PPCP education. Our findings indicate that most CMM essential functions for the two core domains of content and competence can be covered in about 40 hours of direct curricular time and with proper formative assessment and feedback virtually all students can show competence 
with CMM essential functions. These results and the process used to analyze and assess CMM should be valuable to other colleagues seeking to assess their own curriculum.

$\mathrm{CMM}$ is aptly termed comprehensive and failing to commit adequate faculty resources and curricular time for feedback and deliberative practice may compromise student competency. Our analyses provide metrics for colleges planning to implement CMM and found that 41 formal curricular hours devoted to CMM addressed about $70 \%$ of the essential functions. Faculty and student assessment time burden were substantially more than lecture hours with over three times as many course hours devoted to formative and summative assessment of learning than to lecture. Faculty full-time equivalent (FTE) was not quantified, but faculty reported that a SOAP note assignment required about 40 to 60 hours of grading time for 70 students and that individualized faculty feedback for presentations, reassessment, and OCSEs made CMM more labor intensive than other parts of the curriculum. As the block system used at RUHS COP allows students to focus on a single subject at a time, institutions with traditional semester-based classes would need to consider the time students spend outside of class understanding, analyzing and creating assignments when planning schedules with concomitant courses.

Mapping CMM curriculum with essential functions can uncover gaps in teaching, learning and assessment. This study assessed content fidelity by evaluating the degree to which the curriculum aligned with a nationally accepted CMM framework and found a relatively high degree of fidelity to the framework. Still it was surprising that almost $30 \%$ of the essential functions remained either unaddressed or deficient, underscoring the importance of internal curriculum validation. Omissions fell primarily within the areas of follow-up. Potential strategies to correct this issue include developing simulation-based curriculum related to follow-up and monitoring that requires students to revisit patients. As time for course instruction rarely increases, making follow-up a priority may require rethinking how CMM is presented. However, essential functions related to appropriate patient follow-up may be better addressed during experiential rotations where follow-up encounters occur more frequently. Finding the best strategies to incorporate follow-up merits further investigation.

Addressing essential function deficiencies might be more challenging. The intricacy of patient care makes it difficult to recreate the complexity of real-world interactions in a simulation-based learning experience. Similarly, condensed classroom learning experiences may fail to fully capture the nuances of live patient care such as cultural impact. Together omissions and deficiencies underscore opportunities for research investigations on improving CMM curriculum and highlight opportunities for growth and course development.

If pharmacy schools teach CMM but students fail to master it, then fidelity to the "process component" is not achieved. Testing and assignments are the major tools to assess competency. Testing can take many forms: written, multiple choice, or use of simulation. Skills courses require assessment rubrics that directly measure and align with the subject matter. Rubrics closely aligned with CMM help ensure reliability from year to year in teaching and assessment. After developing rubrics, the passing bar must be set at some level to ensure competency. Our results indicate that almost $99 \%$ of students demonstrated competency at a $90 \%$ level by the end of the course, suggesting that CMM can be mastered at the level taught before entering experiential rotations. However, to achieve this overall level of success some students needed an opportunity to remediate and reassess.

Teaching CMM helps prepare pharmacy students to deliver consistent patient care during their APPEs and at a level on par with preceptor expectations. Most students considering higher-level practices such as prescribing will need to seek residency training and obtain board certifications. The CMM process described here helps sets the stage for residency programs and board certifications to further enhance and refine these practices. This is in-line with ACCP's position statement that undergraduate pharmacy education should prepare students to enter pharmacy residency training. ${ }^{19}$

Theoretical barriers to CMM competency include a lack of faculty expertise in CMM, familiarity with the detailed CMM framework and allotting sufficient time for it in an already full curriculum. It was reassuring to find that almost 99\% of students achieved competency, suggesting that with a relatively small investment of curricular time students can master CMM basics in a low stake, low risk environment and subsequently use their clinical experiences to hone their CMM skills during APPEs.

However, there are some limitations to consider before applying these findings to other institutions. First, the study looked at two of the three core CMM elements in one longitudinal patient care course. Also, the study used the four assessments and student competency on these assessments as a proxy to evaluate process fidelity such as quality and student responsiveness. For PPCP others have developed more detailed methods of evaluation. ${ }^{17}$ Potentially critical parts of the framework viewed as omitted or deficient in this study might be presented elsewhere in the curriculum resulting in an underestimation of time. Another limitation was the lack of long-term follow-up in a classroom setting. Additionally, this study did not explore whether CMM training might work better by being more fully integrated throughout the curriculum or split among other courses such a social and administrative science courses where the context domain (not evaluated in this study) could be fully realized. This study did not explore how students applied CMM skills in their 
advanced clinical rotations nor its impact on patient care. These questions suggest the need for future research about educational strategies to promote the CMM framework including its integration into experiential training and ultimately if CMM training translates into better trained pharmacists and improved patient outcomes. Additionally, these results represent a single institution and may not be fully generalizable to other pharmacy programs.

\section{CONCLUSION}

CMM offers a "whole person" method that requires an individualized patient approach using evidence-based management. Promoting fidelity with CMM in pharmacy education is crucial to the advancement of the pharmacy profession. This study found that about $70 \%$ of CMM essential functions for the two core domains of content and competence can be covered and mastered by students in 41.5 hours of direct curricular time. Identified omissions and deficiencies in the curriculum highlight opportunities for future investigations to improve CMM curriculum.

\section{ACKNOWLEDGMENTS}

We would like to acknowledge Dr. Sorensen for his initial review and suggestions on this manuscript.

\section{REFERENCES}

1. Sorensen TD, Hager KD, Schlichte A, Janke K. A dentist, pilot, and pastry chef walk into a bar. . .why teaching PPCP is not enough. Am J Pharm Educ. 2020;84(4): Article 7704.

2. Joint Commission of Pharmacy Practitioners. Pharmacists' Patient Care Process. May 29, 2014. http://www.pharmacist.com/sites/default/files/files/PatientCareProcess.pdf. Accessed April 11, 2021.

3. Roth McClurg M, Sorensen TD, Carroll J, et al. The Patient Care Process for Delivering Comprehensive Medication Management (CMM): Optimizing medication use in patient-centered, team-based care settings. $C$ Primary Care Res Team. 2018. https://www.pcpcc.org/sites/default/files/eventattachments/CMM_Care_Process.pdf Accessed April 11, 2021.

4. Livet M, Blanchard C, Frail C, Sorensen T, McClurg MR. Ensuring effective implementation: a fidelity assessment system for comprehensive medication management. J AM Coll Clin Pharm. 2020;3:57-67. DOI: $10.002 / \mathrm{jac} 5.1155$.

5. O’Donnell. Defining, Conceptualizing, and Measuring Fidelity of Implementation and Its Relationship to Outcomes in K-12 Curriculum Intervention Research. Rev Educ Res. 2008;78(1):33-84. https://doi.org/10.3102/0034654307313793

6. Weiss MJ, Bloom HS, Brock T. A Conceptual framework for studying the sources of variation in program effects. MDRC Working Papers on Research Methodology. https://www.mdrc.org/sites/default/files/ImplementationFidelity.pdf Accessed April 11, 2021.

7. Mowbray CT, Holter MC, Teague GB, Bybee D. Fidelity criteria: development, measurement, and validation. Am J Eval. 2003;24(3):315-340.

8. Hepler CD, Strand LM. Opportunities and responsibilities in pharmaceutical care. Am J Hosp Pharm. 1990;47:533-543.

9. Sanchez AM. Teaching patient-centered care to pharmacy students. Int J Clin Pharm. 2011;33(1):55- 57.

10. Accreditation Council for Pharmacy Education. Accreditation standards and key elements for the professional program in pharmacy leading to the doctor of pharmacy degree. Standards 2016. https://www.acpeaccredit.org/pdf/Standards2016FINAL.pdf Accessed April 26, 2021.

11. American Nurses Association. The Nursing Process. https://www.nursingworld.org/practicepolicy/workforce/what-is-nursing/the-nursing-process/ Accessed April 11, 2021.

12. Lacey K, Pritchett E. Nutrition care process and model: ADA adopts road map to quality care and outcomes management. J Am Diet Assoc. 2003;103(8):1061-1072.

13. American Physical Therapy Association. Guidelines: Physical Therapy Documentation of Patient/Client Management. 2009. https://learningcenter.apta.org/Student/MyCourse.aspx?id=8fd1b5bb-9103-4499-88cdcc422208739c\&ProgramID=dcca7f06-4cd9-4530-b9d3-4ef7d2717b5d

14. Rivkin A. Thinking clinically from the beginning: early introduction of the pharmacists' patient care process. Am J Pharm Educ. 2016;80(10): Article 164.

15. Gonyeau MJ, DiVall M, Conley MP, Lancaster J. Integration of the Pharmacists' Patient Care Process (PPCP) into a comprehensive disease management course Series. Am J Pharm Educ. 2018;82(5):Article 6311.

16. Cooley J, Lee J. An operationalization of the Pharmacists' Patient Care Process at on public college of pharmacy. Am J Pharm Educ. 2017;82(2): Article 6301. 
17. Noureldin M, Melton B. Validity Evidence for a Pharmacists' Patient Care Process Self-Efficacy Scale Among Pharmacy Students. Am J Pharm Educ. 2021;85(2): Article 8290.

18. Kolar C, Hager KD, Losinski V. Beyond the pharmacists' patient care process: cultivating patient care practitioners by utilizing the pharmaceutical care framework. Inov Pharm. 2017;8(3): Article 7.

19. Hester EK, McBane SE, Bottorff MB, Carnes TA, Dell K, Gonyeau MJ, et al. Educational outcomes necessary to enter pharmacy residency training. Pharmacotherapy. 2014;34(4):e22-e25. DOI: 10.1002/phar.1411.

Table 1. Abbreviated CMM Curriculum by Block for Pharmacist Patient Care Process II (PPCP II) Course

Block One

Patient Interviewing and Chart Findings

Identification of Medication Related Problems - Indication, Effectiveness, Safety and Adherence (IESA)

Use of LexiComp (IESA)

Use of Guidelines (IESA)

Development of a Problem List (IESA)

Ordering Disease States \& Setting Goals

Assessing Patient Problems

Collect, Assess, and Plan (CAP) Activity (focus on indication, safety and effectiveness)

Problem-solving using PPCP/Subjective, Objective, Assessment and Plan (SOAP)

SOAP Note and Problem List (PL) Rubric

Reviewing a Medical Record

Medical Record based SOAP Note \& PL assignment ${ }^{\mathrm{a}}$

Block Two

Community based (IPPE patient) SOAP Note \& PL project ${ }^{\mathrm{a}}$

Block Three

Presenting a Patient

Objective Structured Clinical Examination (OSCE) Presenting a Patient ${ }^{\mathrm{a}}$

Block Four

SBAR Communication

OSCE Patient interview, SOAP note, \& $\mathrm{PL}^{\mathrm{b}}$

\footnotetext{
${ }^{a}$ Formative assessment of learning
}

${ }^{\mathrm{b}}$ Summative assessment of learning 
Table 2. Content Fidelity: Mapping Course to CMM Essential Functions 1, 3, 4, and $5^{\text {a }}$

\begin{tabular}{|c|c|c|c|}
\hline \multicolumn{4}{|c|}{ Content } \\
\hline \multirow[b]{2}{*}{ Essential Functions } & \multirow[b]{2}{*}{ Curriculum Activities } & \multicolumn{2}{|c|}{ Competency } \\
\hline & & $\begin{array}{c}\text { Formative Assessment of } \\
\text { Learning }\end{array}$ & $\begin{array}{c}\text { Summative } \\
\text { Assessment of } \\
\text { Learning }\end{array}$ \\
\hline
\end{tabular}

Collect and Analyze

Information

1a. Conduct a review of the medical record

1b. Preparation for and conduction of a comprehensive review of medications

1c. Analyze information in preparation for formulating an assessment of medication therapy problems
Completed as a part of essential function 2 and incorporated into teaching of the Development of a Problem List (IESA) lecture
Patient Interviewing and Chart Findings

Identification of Medication Related Problems - Indication, Effectiveness, Safety and Adherence (IESA) Use of LexiComp (IESA) Use of guidelines (IESA)

Reviewing a Medical Record

Community based (IPPE patient) SOAP Note and Problem List (PL) project

3b. Monitoring parameters (IESA)

\section{Develop the Care Plan}

3a. Patient and Provider

Collaboration

(2)

Collect, Assess, and Plan

(CAP) Activity

Use of LexiComp (IESA)

Development of a Problem

List (IESA)

SOAP Note and Problem List

(PL) Rubric

3c. Accurate and updated medication list

3d. Coordinate care plan
Patient Interviewing and Chart Findings SOAP Note and Problem List (PL) Rubric Collect, Assess and Plan (CAP) Activity

SOAP Note and Problem List (PL) Rubric
Community based (IPPE patient) SOAP note \& PL project

Community based (IPPE patient) SOAP note \& PL project

Medical Record based SOAP

Note and PL assignment

Community based (IPPE patient) SOAP note \& PL project

Medical Record based SOAP

Note and PL assignment

Completed as a part of essential function 2 and incorporated into teaching of the Development of a Problem List (IESA) lecture
Medical Record based SOAP Note and PL assignment

OSCE Patient

interview, SOAP note, \& PL (for 1a-

1b)

Completed as a part of essential function 2. Incorporated into Development of a Problem List (IESA) lecture

Community based (IPPE patient) SOAP note \& PL project

Medical Record based SOAP

Note and PL assignment (for

OSCE Patient

interview, SOAP note, \& PL (for 3a$3 \mathrm{~g})$ 
3e. Follow up (what type?)

3f. Follow up (timeframe?)

3g. Determine the appropriate

mode for follow up

\section{Implement the Care Plan $^{\text {a }}$}

4c. Patient education on

disease, medication, and

lifestyle

Collect, Assess, and Plan

(CAP) Activity

(for 3d-3g) and Plan (CAP) Activity 4d. Provide the patient with an updated, accurate medication list

4e. Implement pharmacist recommendations

4f. Communicate care plan

4g. Document encounter in health record

4h. Arrange patient follow-up

4i. Communicate instructions for follow-up with the patient
SOAP Note and Problem List (PL) Rubric Collect, Assess,

SOAP Note and Problem List (PL) Rubric

SOAP Note and Problem List (PL) Rubric Collect, Assess, and Plan (CAP) Activity

SOAP Note and Problem List (PL) Rubric Collect, Assess, and Plan (CAP) Activity Presenting a Patient SBAR Communication

SOAP Note and Problem List (PL) Rubric

SOAP Note and Problem List (PL) Rubric Collect, Assess, and Plan (CAP) Activity

SOAP Note and Problem List (PL) Rubric Collect, Assess, and Plan (CAP) Activity
Community based (IPPE patient) SOAP note \& PL project

Medical Record based SOAP

Note and PL assignment (for $4 c-4 e$ )
OSCE Patient

interview, SOAP note, \& PL
Community based (IPPE patient) SOAP note \& PL project Medical Record based SOAP Note and PL assignment OSCE Presenting a Patient

Community based (IPPE patient) SOAP note \& PL project Medical Record based SOAP Note and PL assignment (for 4g-4i)

Abbreviations: PL (problem list), OSCE (objective structured clinical examination), SOAP (subjective, objective, assessment, and plan), IPPE (introductory pharmacy practice experience), IESA (indication, effectiveness, safety and adherence)

${ }^{a}$ Missing essential functions $4 \mathrm{a}, 4 \mathrm{~b}, 5 \mathrm{a}, 5 \mathrm{~b}$, and $5 \mathrm{c}$ 
Table 3. Content Fidelity: Omissions and Deficiencies in Curriculum

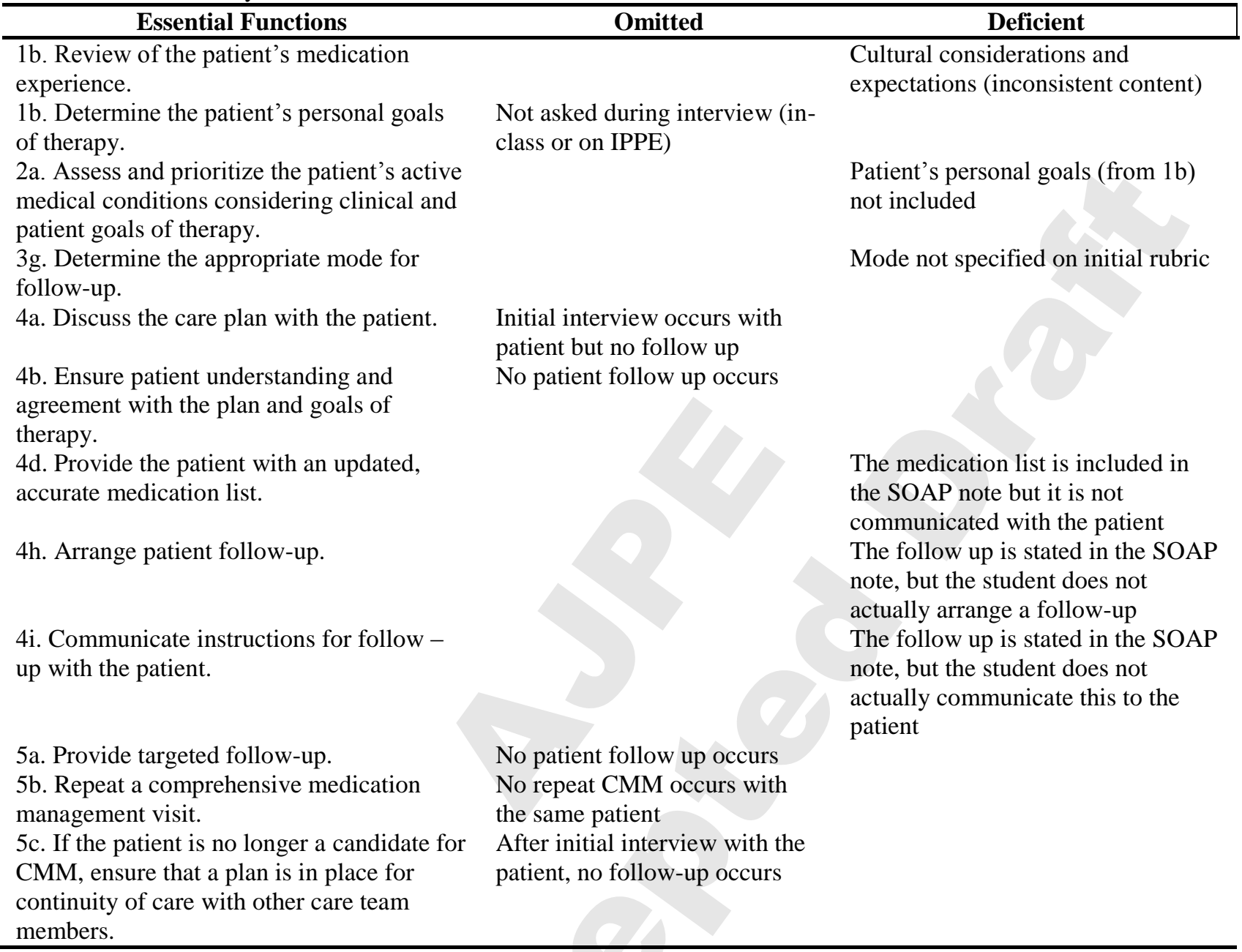


Table 4. Competency Fidelity: Formative Assessment of Learning

\begin{tabular}{|c|c|c|c|}
\hline \multirow[b]{2}{*}{ Academic Year } & \multicolumn{3}{|c|}{ Formative Assessment of Learning } \\
\hline & $\begin{array}{l}\text { Medical Record } \\
\text { based SOAP note } \\
\text { \& PL assignment }\end{array}$ & $\begin{array}{c}\text { Community } \\
\text { based (IPPE } \\
\text { patient) SOAP } \\
\text { Note \& PL } \\
\text { project } \\
\end{array}$ & $\begin{array}{l}\text { OSCE Presenting } \\
\text { a Patient }\end{array}$ \\
\hline \multicolumn{4}{|l|}{$2018-19$} \\
\hline $\begin{array}{l}\% \text { mean score first attempt (\# of } \\
\text { students in course) }\end{array}$ & $85.6 \%(81)$ & $93.1 \%(81)$ & $96.6 \%(80)^{\mathrm{a}}$ \\
\hline Min score & $50 \%$ & $10 \%$ & $75 \%$ \\
\hline Max score & $95 \%$ & $100 \%$ & $100 \%$ \\
\hline \# students needing remediation & 8 & 7 & 2 \\
\hline \multicolumn{4}{|l|}{$\%$ and ratio achieving competency } \\
\hline First Attempt & $90.1 \%(73 / 81)$ & $91.4 \%(74 / 81)$ & $97.5 \%(78 / 80)$ \\
\hline $\begin{array}{l}\text { Second Attempt (after } \\
\text { remediation) }\end{array}$ & $100 \%(81 / 81)$ & $100 \%(81 / 81)$ & $98.8 \%(79 / 80)$ \\
\hline \multicolumn{4}{|l|}{ 2019-20 } \\
\hline $\begin{array}{l}\% \text { mean score prior to remediation } \\
\text { (\# of students in course) }\end{array}$ & $86.7 \%(77)$ & $88.1 \%(77)$ & $94.2 \%(78)^{\mathrm{a}}$ \\
\hline Min score & $63 \%$ & $54.5 \%$ & $90.3 \%^{\mathrm{b}}$ \\
\hline Max score & $97 \%$ & $100 \%$ & $100 \%$ \\
\hline \# students remediating & 9 & 18 & 1 \\
\hline \multicolumn{4}{|l|}{$\%$ and ratio achieving competency } \\
\hline First Attempt & $88.3 \%(68 / 77)$ & $76.6 \%(59 / 77)$ & $98.7 \%(77 / 78)$ \\
\hline $\begin{array}{l}\text { Second Attempt (after } \\
\text { remediation) }\end{array}$ & $100 \%(77 / 77)$ & $100 \%(77 / 77)$ & $98.7 \%(77 / 78)$ \\
\hline 2018-2020 Combined & & & \\
\hline $\begin{array}{l}\text { Total \% student achieving } \\
\text { competency }\end{array}$ & $100 \%(158 / 158)$ & $100 \%(158 / 158)$ & $98.7 \%(156 / 158)$ \\
\hline
\end{tabular}

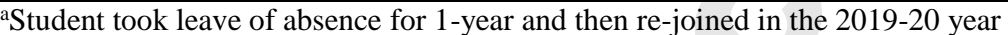

${ }^{\mathrm{b}}$ One student received a score of 0 , this number was excluded here to better show the score spread 
Table 5. Competency Fidelity: Summative Assessment of Learning

\begin{tabular}{|c|c|c|c|}
\hline \multirow[b]{2}{*}{ Academic Year } & \multicolumn{3}{|c|}{ Summative Assessment of Learning } \\
\hline & $\begin{array}{c}\text { OSCE Patient } \\
\text { interview, SOAP note, } \\
\text { \& PL First Attempt }\end{array}$ & $\begin{array}{c}\text { OSCE Patient } \\
\text { interview, SOAP } \\
\text { note, \& PL Second } \\
\text { Attempt } \\
\end{array}$ & $\begin{array}{c}p \text {-value Test } \\
\text { and retest }\end{array}$ \\
\hline \multicolumn{4}{|l|}{$2018-19$} \\
\hline$\%$ mean score (\# of students in course) & $92.2 \%(80)$ & $94.7 \%(80)$ & $0.004^{\mathrm{a}}$ \\
\hline Min score & $58.3 \%$ & $85 \%$ & \\
\hline Max score & $100 \%$ & $100 \%$ & \\
\hline \# students needing remediation & 10 & $1^{\mathrm{c}}$ & \\
\hline $\begin{array}{l}\% \text { and ratio achieving competency } \\
\text { First Attempt } \\
\text { Second Attempt (After remediation) }\end{array}$ & $87.5 \%(70 / 80)$ & $98.8 \%(79 / 80)$ & \\
\hline \multicolumn{4}{|l|}{$2019-20$} \\
\hline$\%$ mean score (\# of students in course) & $86.4 \%(78)$ & $91.6 \%(78)$ & $<0.01^{\mathrm{a}}$ \\
\hline Min score & $28.6 \%$ & $48.6 \%$ & \\
\hline Max score & $97.1 \%$ & $97.1 \%$ & \\
\hline \# students needing remediation & 24 & $1^{\mathrm{b}}$ & \\
\hline \multicolumn{4}{|l|}{$\%$ and ratio achieving competency } \\
\hline First Attempt & $69.2 \%(54 / 78)$ & & \\
\hline Second Attempt (After remediation) & & $98.7 \%(77 / 78)$ & \\
\hline 2018-20 Combined & & & \\
\hline $\begin{array}{l}\text { Overall \% and ratio of student } \\
\text { competency }\end{array}$ & $78.5 \%(124 / 158)$ & $98.7 \%(156 / 158)$ & \\
\hline
\end{tabular}

a\% mean score comparisons calculated using two-tailed, paired t-test; comparison of students achieving competency by the end of the course using Chi-square; all $p$-values $<0.05$ are significant

${ }^{b}$ One student each year did not pass summer remediation and repeated course 